\title{
4
}

\section{High Throughput Transmissions in OFDM based Random Access Wireless Networks}

\author{
Nuno Souto1,2, Rui Dinis',3, João Carlos Silva1,2, \\ Paulo Carvalho ${ }^{3}$ and Alexandre Lourenço ${ }^{1,2}$ \\ IISCTE-IUL \\ 2Instituto de Telecomunicações, \\ ${ }^{3}$ UNINOVA/FCT-UNL, \\ Portugal,
}

\section{Introduction}

In Random Access Wireless Networks it is common to occur packet collisions due to different users trying to access simultaneously to a given physical channel. The conventional approach is to discard all blocks involved in the collision and retransmit them again. To reduce the chances of multiple collisions each user transmits in the next available slot with a given probability. With this strategy, if two packets collide we need at least three time slots to complete the transmission (more if there are multiple collisions), which results in a throughput loss.

To overcome this problem, a TA (Tree Algorithm) combined with a SIC (Successive Interference Cancellation) scheme was proposed in (Yu \& Giannakis, 2005). Within that scheme, the signal associated to a collision is not discarded. Instead, if the packets of two users collide then, once we receive with success the packet of one of those users, we can subtract the corresponding signal from the signal with collision and recover the packet from the other user. With this strategy, a collision involving two packets requires only one additional time slot to complete the transmission, unless there are multiple collisions. However, the method has a setback since possible decision errors might lead to a deadlock. (Wang et al., 2005) Another problem with these techniques is that we do not take full advantage of the information in the collision. The ideal situation would be to use the signals associated to multiple collisions to separate the packets involved (in fact, solving collisions can be regarded as a multiuser detection problem). In (Tsatsanis et al., 2000) a multipacket detection technique was proposed where all users involved in a collision of $N_{P}$ packets retransmit their packets $N_{P}-1$ times, each one with a different phase rotation to allow packet separation. However, this technique is only suitable for flat-fading channels (there are phase rotations that might lead to an ill-conditioned packet separation). Moreover, it is difficult to cope with channel variations during the time interval required to transmit the $N_{P}$ variants of each packets (the same was also true for the SIC-TA technique of (Yu \& Giannakis, 2005). A variant of these techniques suitable for time-dispersive channels was proposed in (Zhang \& Tsatsanis, 2002) although the receiver complexity can become very high for severely timedispersive channels.

Source: Communications and Networking, Book edited by: Jun Peng,

ISBN 978-953-307-114-5, pp. 434, September 2010, Sciyo, Croatia, downloaded from SCIYO.COM 
A promising method for resolving multiple collisions was proposed in (Dinis, et al., 2007) for SC modulations (Single Carrier) with FDE (Frequency-Domain Equalization). Since that technique is able to cope with multiple collisions, the achievable throughputs can be very high (Dinis, et al., 2007). In this chapter we extend that approach to wireless systems employing OFDM modulations (Orthogonal Frequency Division Multiplexing) (Cimini, 1985), since they are currently being employed or considered for several digital broadcast systems and wireless networks (Nee \& Prasad, 2000) (3GPP TR25.814, 2006). To detect all the simultaneously transmitted packets we propose an iterative multipacket receiver capable of extracting the packets involved in successive collisions. The receiver combines multipacket separation with interference cancellation (IC). To be effective our receiver requires uncorrelated channels for different retransmissions. Therefore, to cope with quasi-stationary channels, different interleaved versions of the data blocks are sent in different retransmissions.

In this chapter it is also given some insight into the problem of estimating the number of users involved in a collision by analyzing the probability distribution of the decision variable and selecting a convenient detection threshold. The problem of estimating the channel characteristics (namely the channel frequency response) of each user is also addressed. Regarding this issue and due to its iterative nature the proposed receiver can perform enhanced channel estimation.

The chapter is organized as follows. First the system model is defined in Section 2 while Section 3 and 4 describe the proposed transmitter and multipacket receiver in detail. The MAC scheme is analyzed in Section 5 while Section 6 presents some performance results. Finally the conclusions are given on Section 7.

\section{System description}

In this chapter we consider a random access wireless network employing an OFDM scheme with $N$ subcarriers where each user can transmit a packet in a given time slot. If $N_{p}$ users decide to transmit a packet in the same time slot then a collision involving $N_{p}$ packets will result. In this case, all packets involved in the collision will be retransmitted $N_{p}-1$ times. In practice, the receiver (typically the BS - Base Station) just needs to inform all users of how many times they have to retransmit their packets (and in which time-slots, to avoid collisions with new users).The request for retransmissions can be implemented very simply with a feedback bit that is transmitted to all users. If it is a ' 1 ' any user can try to transmit in the next time slot. When it becomes ' 0 ' the users that tried to transmit in the last time slot must retransmit their packets in the following time slots until the bit becomes a ' 1 '. All the other users cannot transmit any packet while the bit is ' 0 '.

The receiver detects the packets involved in the collision as soon as it receives $N_{p}$ different signals associated to the collision of the $N_{p}$ packets. The figure (Fig. 1) illustrates the sequence of steps using an example with 2 users.

At the receiver, the basic idea is to use all these received transmission attempts to separate the $N_{p}$ colliding packets. In fact, our system can be regarded as a MIMO system (MultipleInput, Multiple Output) where each input corresponds to a given packet and each output corresponds to each version of the collision. To accomplish a reliable detection at the receiver it is important that the correlation between multiple received retransmissions (i.e., multiple versions of each packet involved in the collision) is a low as possible. For static or slow-varying channels this correlation might be very high, unless different frequency bands 


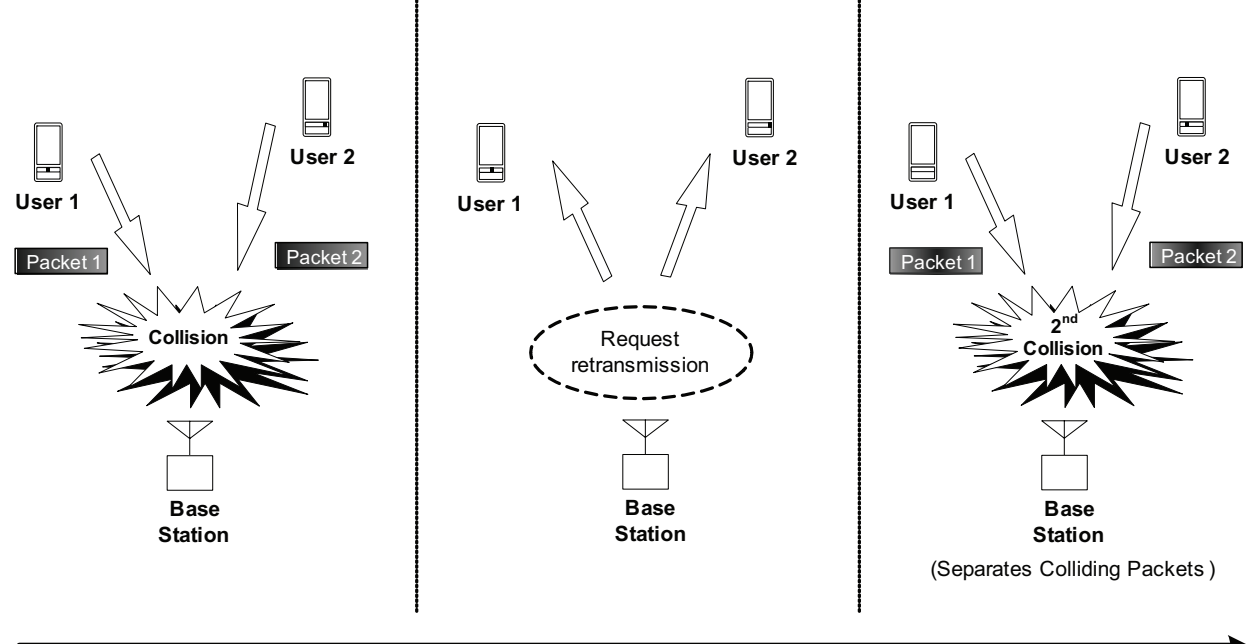

Time

Fig. 1. Sequence of steps required for the multipacket detection method for the case of 2 colliding packets.

are adopted for each retransmission. To overcome this problem, we can take advantage of the nature of OFDM transmission over severely time-dispersive channels where the channel frequency response can change significantly after just a few subcarriers. This means that the channel frequency response for subcarriers that are not close (i.e., subcarriers in different parts of the OFDM band) can be almost uncorrelated. Therefore, by simply applying a different interleaving to the modulated symbols in each retransmission it is possible to reduce the correlation between them ${ }^{1}$. In this chapter we will call them symbol interleavers to distinguish from the other interleaving blocks²).

\section{Transmitter design}

In Fig. 2 it is shown the block diagram representing the processing chain of a transmitter designed to be used with the proposed packet separation scheme.

According to the diagram the information bits are first encoded and rate matching is applied to fit the sequence into the radio frame, which is accomplished by introducing or removing bits. The resulting encoded sequence is interleaved and mapped into complex symbols according to the chosen modulation. A selector then chooses to apply a symbol interleaver or not depending on whether it is a retransmission or the first transmission attempt. A total

\footnotetext{
1 Clearly, using different symbol-level interleavers before mapping the coded symbols in the OFDM subcarriers is formally equivalent to interleave the channel frequency response for different subcarriers. For a given subcarrier, this reduces the correlation between the channel frequency response for different retransmissions.

2 It should be pointed out that in this chapter we assume that the interleaver to reduce the correlation between different retransmissions operates at the symbol level and the interleavers associated to the channel encoding are at the bit level. However, all interleavers could be performed at the bit level.
} 


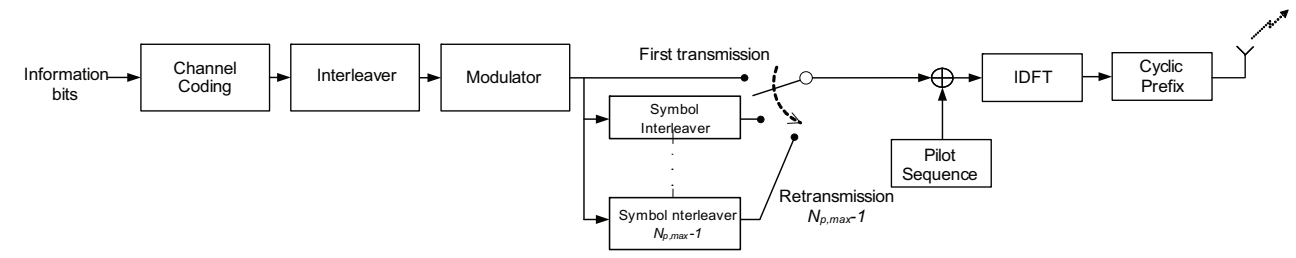

Fig. 2. Emitter Structure

of $N_{p, \max }-1$ different symbol interleavers are available, where $N_{p, \max }$ is the maximum number of users that can try to transmit simultaneously, so that a different one is applied in each retransmission. Known pilot symbols are inserted into the modulated symbols sequence before the conversion to the time domain using an IDFT (Inverse Discrete Fourier Transform). As will be explained further ahead, the pilot symbols are used for accomplishing user activity detection and channel estimation at the base station.

\section{Receiver design}

\subsection{Receiver structure}

To detect the multiple packets involved in a collision we propose the use of an iterative receiver whose structure is shown in Fig. 3.

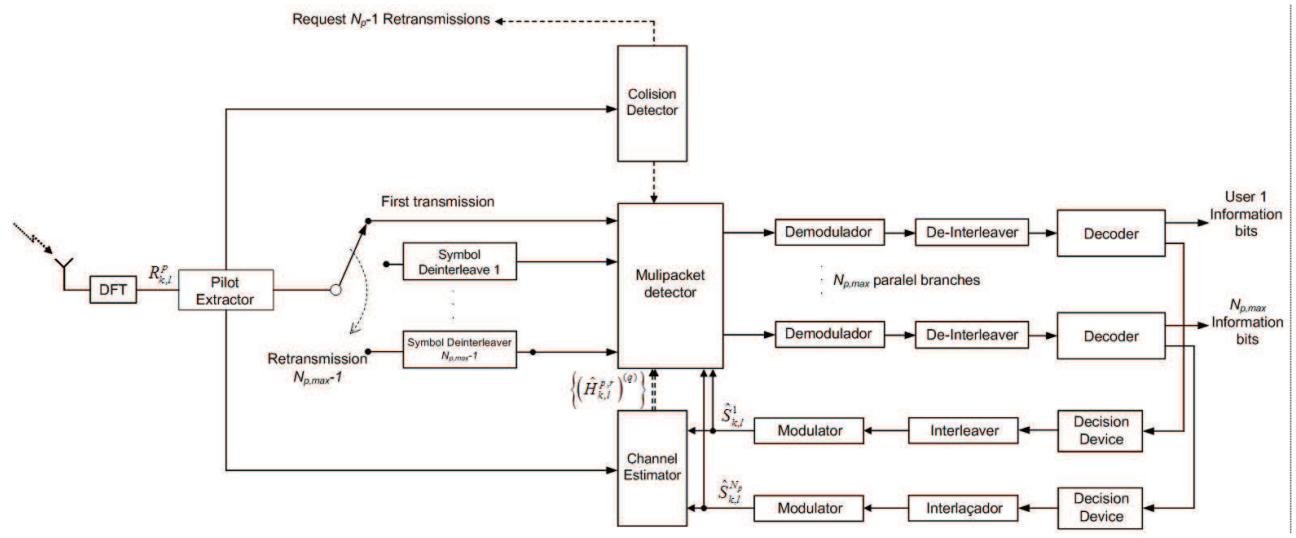

Fig. 3. Iterative receiver structure.

For simplicity we will assume that different packets arrive simultaneously. In practice, this means that some coarse time-advance mechanism is required, although some residual time synchronization error can be absorbed by the cyclic prefix. As with other OFDM-based schemes, accurate frequency synchronization is also required. First, the received signals corresponding to different retransmissions, which are considered to be sampled and with the cyclic prefix removed, are converted to the frequency domain with an appropriate size$N$ DFT operation. Pilot symbols are extracted for user activity detection in the "Collision Detection" block as well as for channel estimation purposes while the data symbols are deinterleaved according to the retransmission to which they belong.

Assuming that the cyclic prefix is longer than the overall channel impulse response (the typical situation in OFDM-based systems) the resulting sequence for the $r^{\text {th }}$ transmission attempt can be written as: 


$$
R_{k, l}^{r}=\sum_{p=1}^{N_{p}} S_{k, l}^{p} H_{k, l}^{p, r}+N_{k, l}^{r}
$$

with $H_{k, l}^{p, r}$ denoting the overall channel frequency response in the $k^{\text {th }}$ frequency of the $l^{\text {th }}$ OFDM block for user $p$ during transmission attempt $r . N_{k, l}^{p}$ denotes the corresponding channel noise and $S_{k, l}^{p}$ is the data symbol selected from a given constellation, transmitted on the $k^{\text {th }}(k=1, \ldots, N)$ subcarrier of the $l^{\text {th }}$ OFDM block by user $p\left(p=1, \ldots, N_{p}\right)$. Since we are applying interleaving to the retransmissions, to simplify the mathematical representation we will just assume that it is the sequence of channel coefficients $H_{k, l}^{p, r}$ that are interleaved instead of the symbols (therefore we do not use the index $r$ in $S_{k, l}^{p}$ ).

After the symbol de-interleavers the sequences of samples associated to all retransmissions are used for detecting all the packets inside the Multipacket Detector with the help of a channel estimator block. After the Multipacket Detector, the demultiplexed symbols sequences pass through the demodulator, de-interleaver and channel decoder. This channel decoder has two outputs: one is the estimated information sequence and the other is the sequence of log-likelihood ratio (LLR) estimates of the code symbols. These LLRs are passed through the Decision Device which outputs soft-decision estimates of the code symbols. These estimates enter the Transmitted Signal Rebuilder which performs the same operations of the transmitters (interleaving, modulation). The reconstructed symbol sequences are then used for a refinement of the channel estimates and also for possible improvement of the multipacket detection task for the subsequent iteration. This can be accomplished using an IC in the Multipacket Detector block.

\subsection{Multipacket Detector}

The objective of the Multipacket Detector is to separate multiple colliding packets. It can accomplish this with several different methods. In the first receiver iterations it can apply either the MMSE criterion (Minimum Mean Squared Error), the ZF criterion (Zero Forcing) or a Maximum Likelihood Soft Output criterion (MLSO) (Souto et al., 2008). Using matrix notation the MMSE estimates of the transmitted symbols in subcarrier $k$ and OFDM block $l$ is given by

$$
\hat{\mathbf{S}}_{k, l}=\hat{\mathbf{H}}_{k, l}{ }^{H} \cdot\left(\hat{\mathbf{H}}_{k, l} \hat{\mathbf{H}}_{k, l}{ }^{H}+\sigma^{2} \mathbf{I}\right)^{-1} \mathbf{R}_{k, l}
$$

where $\hat{\mathbf{S}}_{k, l}$ is the $N_{p} \times 1$ estimated transmitted signal vector with one user in each position, $\hat{\mathbf{H}}_{k, l}$ is the $N_{p} \times N_{p}$ channel matrix estimate with each column representing a different user and each line representing a different transmission attempt, $\mathbf{R}_{k, l}$ is the $N_{p} \times 1$ received signal vector with one received transmission attempt in each position and $\sigma^{2}$ is the noise variance. The ZF estimate can be simply obtained by setting $\sigma$ to 0 in (2). In the MLSO criterion we use the following estimate for each symbol

$$
\hat{S}_{k, l}^{p}=E\left[S_{k, l}^{p} \mid \mathbf{R}_{k, l}\right]=\sum_{s_{i} \in \Lambda} s_{i} \cdot \frac{P\left(S_{k, l}^{p}=s_{i}\right)}{p\left(\mathbf{R}_{k, l}\right)} p\left(\mathbf{R}_{k, l} \mid S_{k, l}^{p}=s_{i}\right)
$$


where $s_{i}$ corresponds to a constellation symbol from the modulation alphabet $\Lambda, E[\cdot]$ is the expected value, $P(\cdot)$ represents a probability and $p(\cdot)$ a probability density function (PDF). Considering equiprobable symbols $P\left(S_{k, l}^{p}=s_{i}\right)=1 / M$, where $M$ is the constellation size. The PDF values required in (3) can be computed as:

$$
\begin{gathered}
p\left(\mathbf{R}_{k, l} \mid S_{k, l}^{p}=s_{i}\right)=\frac{1}{M^{N_{p}-1}} \sum_{\mathbf{S}_{k, l}^{\text {interf }} \in \Lambda^{N_{p}-1}} p\left(\mathbf{R}_{k, l} \mid S_{k, l}^{p}=s_{i}, \mathbf{S}_{k, l}^{\text {interf }}\right) \\
=\frac{1}{M^{N_{p}-1}} \sum_{S_{k, l}^{\text {interf }} \in \Lambda^{N_{p}-1}} \frac{1}{\left(2 \pi \sigma^{2}\right)^{N_{p}}} \exp \left[\sum_{r=1}^{N_{p}}-\frac{\left.\left|R_{k, l}^{r}-\sum_{m=1}^{N_{p}} S_{k, l}^{m} \hat{H}_{k, l}^{m, r}\right|^{2}\right]}{2 \sigma^{2}}\right]
\end{gathered}
$$

Where $\mathbf{S}_{k, l}^{\text {interf }}$ is a $\left(N_{p}-1\right) \times 1$ vector representing a possible combination of colliding symbols except the one belonging to packet $p$. An interference canceller (IC) can also be used inside the Multipacket Detector, but usually is only recommendable after the first receiver iteration (Souto et al., 2008). In iteration $q$, for each packet $p$ in each transmission attempt $r$, the IC subtracts the interference caused by all the other packets in that attempt. This can be represented as:

$$
\left(R_{k, l}^{r, p}\right)^{(q)}=R_{k, l}^{r}-\sum_{\substack{m=1 \\ m \neq p}}^{N_{p}}\left(\hat{S}_{k, l}^{m}\right)^{(q-1)} \hat{H}_{k, l}^{m, r}
$$

Where $\left(\hat{S}_{k, l}^{m}\right)^{(q-1)}$ is the transmitted symbol estimate obtained in the previous iteration for packet $m$, subcarrier $k$ and OFDM block $l$.

\subsection{Channel estimation}

To achieve coherent detection at the receiver known pilot symbols are periodically inserted into the data stream. The proposed frame structure is shown in Fig. 4. For an OFDM system with $N$ carriers, pilot symbols are multiplexed with data symbols using a spacing of $\Delta N_{T}$ OFDM blocks in the time domain and $\Delta N_{F}$ subcarriers in the frequency domain. To avoid interference between pilots of different users, FDM (Frequency Division Multiplexing) is employed for the pilots, which means that pilot symbols cannot be transmitted over the same subcarrier by different users. No user can transmit data symbols on subcarriers reserved for pilots, therefore, the minimum allowed spacing in the frequency domain is $\left(\Delta N_{F}\right)_{\min }=N_{p, \max }$, where $N_{p, \max }$ is the maximum number of users that can try to transmit simultaneously.

To obtain the frequency channel response estimates for each transmitting/receiving antenna pair the receiver applies the following steps in each iteration: 

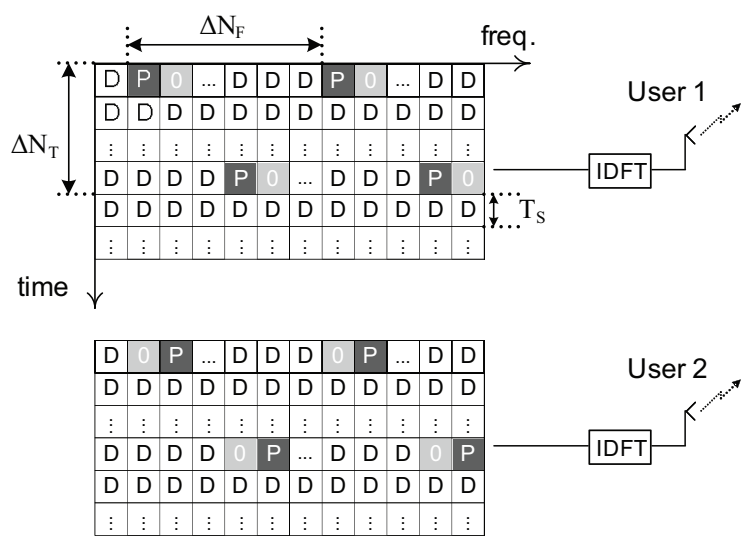

Fig. 4. Proposed frame structure for MIMO-OFDM transmission with implicit pilots (P pilot symbol, D - data symbol, 0 - empty subcarrier).

1. The channel estimate between transmit antenna $m$ and receive antenna $n$ for each pilot symbol position, is simply computed as:

$$
\tilde{H}_{k, l}^{p, r}=\frac{\left(S_{k, l}^{p, \text { Pilot }}\right)^{*}}{\left|S_{k, l}^{p, \text { Pilot }}\right|^{2}} R_{k, l}^{r}
$$

where $S_{k, l}^{p, \text { Pilot }}$ corresponds to a pilot symbol transmitted in the $k^{\text {th }}$ subcarrier of the $l^{\text {th }}$ OFDM block by user $p$. Obviously, not all indexes $k$ an $l$ will correspond to a pilot symbol since $\Delta N_{T}>1$ or $\Delta N_{F}>1$.

2. Channel estimates for the same subcarrier $k$, user $p$ and transmission attempt $r$ but in time domain positions (index $l$ ) that do not carry a pilot symbol can be obtained through interpolation using a finite impulse response (FIR) filter with length $W$ as follows:

$$
\tilde{H}_{k, l+t}^{p, r}=\sum_{j=-\lfloor(W-1) / 2\rfloor}^{\lfloor W / 2\rfloor} h_{t}^{j} \tilde{H}_{k, l+j \cdot \Delta N_{T}}^{p, r}
$$

where $t$ is the OFDM block index relative to the last one carrying a pilot (which is block with index $l$ ) and $h_{t}^{j}$ are the interpolation coefficients of the estimation filter which depend on the channel estimation algorithm employed. There are several proposed algorithms in the literature like the optimal Wiener filter interpolator (Cavers, 1991) or the low pass sinc interpolator (Kim et al., 1997).

3. After the first iteration the data estimates can also be used as pilots for channel estimation refinement (Valenti, 2001). The respective channel estimates are computed as 


$$
\left(\tilde{H}_{k, l}^{p, r}\right)^{(q)}=\frac{R_{k, l}^{r}\left(\hat{S}_{k, l}^{p}\right)^{(q-1)^{*}}}{\left|\left(\hat{S}_{k, l}^{p}\right)^{(q-1)}\right|^{2}}
$$

4. The channel estimates are enhanced by ensuring that the corresponding impulse response has a duration $N_{G}$ (number of samples at the cyclic prefix). This is accomplished by computing the time domain impulse response through $\left\{\left(\tilde{h}_{i, l}^{p, r}\right)^{(q)} ; \mathrm{i}=0,1, \ldots, \mathrm{N}-1\right\}=\operatorname{DFT}\left\{\left(\tilde{H}_{k, l}^{p, r}\right)^{(q)} ; \mathrm{k}=0,1, \ldots, \mathrm{N}-1\right\}$, followed by the truncation of this sequence according to $\left\{\left(\hat{h}_{i, l}^{p, r}\right)^{(q)}=w_{i}\left(\tilde{h}_{i, l}^{p, r}\right)^{(q)} ; \mathrm{i}=0,1, \ldots, \mathrm{N}-1\right.$ with $w_{i}=1$ if the $i^{\text {th }}$ time domain sample is inside the cyclic prefix duration and $w_{i}=0$ otherwise. The final frequency response estimates are then obtained as $\left\{\left(\hat{H}_{k, l}^{p, r}\right)^{(q)} ; \mathrm{k}=0,1, \ldots, \mathrm{N}-1\right\}=\operatorname{IDFT}\left\{\left(\hat{h}_{i, l}^{p, r}\right)^{(q)}\right.$; $\mathrm{i}=0,1, \ldots, \mathrm{N}-1\}$.

\subsection{Detection of users involved in a collision}

One of the difficulties of employing multipacket detector schemes, namely the ones proposed in this chapter, lies in finding out which users have packets involved in the collision. Missing a user will result in an insufficient number of retransmissions to reliably extract the others while assuming a non-transmitting user as being active will also degrade the packet separation and waste resources by requesting an excessive number of retransmissions. In the following we propose a simple detection method that can be combined with the multipacket detection approach described previously. This method considers the use of OFDM blocks with pilots multiplexed with conventional data blocks, as described in the previous subsection. We assume that the maximum number of users that can attempt to transmit their packets in a given physical channel is $N_{p, \max }$. Since each user $p$ has a specific subset of subcarriers reserved for its pilot symbols the receiver can use those subcarriers to estimate whether the user is transmitting a packet or not. To accomplish that objective it starts by computing the decision variable:

$$
Y_{p}=\sum_{k^{\prime}, l^{\prime}}^{N_{\text {pilots }}}\left|R_{k^{\prime}, l^{\prime}}^{1}\right|^{2}, \quad p=1, \ldots, N_{p, \max }
$$

for all users, with $\left(k^{\prime}, l^{\prime}\right)$ representing all positions (subcarriers and OFDM blocks) containing a pilot symbol of user $p$ and $N_{\text {pilots }}$ being the total number of pilots used inside the sum. The decision variable, $Y_{p}$, can then be compared with a threshold $y^{\text {th }}$ to decide if a user is active or not.

The threshold should be chosen so as to maximize the overall system throughput. Assuming a worst-case scenario where any incorrect detection of the number of users results in the loss of all packets then, from (Tsatsanis et al., 2000), the gross simplified system throughput (not taking into account bit errors in decoded packets) is given by:

$$
R=\frac{N_{p, \max }\left(1-P_{e}\right)}{N_{p, \max }\left(1-P_{e}\right)+P_{e}^{N_{p, \max }}}\left(1-P_{M}\right)\left[\left(1-P_{e}\right)\left(1-P_{M}\right)+P_{e}\left(1-P_{F}\right)\right]^{N_{p, \max }-1}
$$


where $P_{e}$ is the probability of a user's buffer being empty at the beginning of a transmission slot, $P_{M}$ is the probability of a missed detection and $P_{F}$ is the false alarm probability. The threshold, $y^{\text {th }}$, that maximizes (10) can be found through:

$$
\frac{\partial R}{\partial y}=0
$$

resulting

$$
\frac{\partial P_{M}}{\partial y}\left[\left(1-P_{e}\right)\left(1-P_{M}\right) N_{p, \text { max }}+P_{e}\left(1-P_{F}\right)\right]=\left(N_{p, \max }-1\right)\left(1-P_{M}\right) P_{e} \frac{\partial\left(1-P_{F}\right)}{\partial y}
$$

Assuming low false alarm and missed detection probabilities, i.e.,

$$
\left\{\begin{array}{l}
1-P_{M} \approx 1 \\
1-P_{F} \approx 1
\end{array}\right.
$$

and noting that:

$$
\left\{\begin{array}{l}
p_{1}(y)=\frac{\partial\left(1-P_{F}\right)}{\partial y} \\
p_{2}(y)=\frac{\partial P_{M}}{\partial y}
\end{array}\right.
$$

where $p_{1}(y)$ is the probability density function (PDF) of $\sum_{k^{\prime}, l^{\prime}}^{N_{\text {pilots }}}\left|N_{k^{\prime}, l^{\prime}}^{1}\right|^{2}$ and $p_{2}(y)$ is the PDF of $\sum_{k^{\prime}, l^{\prime}}^{N_{\text {pilots }}}\left|S_{k^{\prime}, l^{\prime}}^{p, p i l o t} H_{k^{\prime}, l^{\prime}}^{p, 1}+N_{k^{\prime}, l^{\prime}}^{1}\right|^{2},(12)$ can be rewritten as

$$
p_{1}(y)=p_{2}(y) \frac{N_{p, \max }-P_{e}\left(N_{p, \max }-1\right)}{\left(N_{p, \max }-1\right) P_{e}}
$$

Therefore we can compute the threshold from the weighted intersection of the two PDFs, $p_{1}(y)$ and $p_{2}(y)$. Regarding the first PDF, since $N_{k, l}^{1}$ are zero mean independent complex Gaussian variables with variance $E\left[\left|N_{k, l}^{1}\right|^{2}\right]=\mathrm{N}_{0} \quad\left(\mathrm{~N}_{0} / 2\right.$ is the noise power spectral density), $\left|N_{k, l}^{1}\right|^{2}$ will have an exponential distribution with average $\mu_{1}=E\left[\left|N_{k, l}^{1}\right|^{2}\right]$. Therefore the decision variable corresponds to a sum of independent exponential random variables and, as a result, follows an Erlang distribution expressed as

$$
p_{1}(y)=\frac{y^{N_{\text {pilots }}-1} \exp \left(-\frac{y}{\mu_{1}}\right)}{\mu_{1}^{N_{\text {pilots }}}\left(N_{\text {pilots }}-1\right) !}
$$


Regarding the second PDF, $R_{k, l}^{1}=S_{k, l}^{p, p i l o t} H_{k, l}^{p, 1}+N_{k, l}^{1}$ and $\left|R_{k, l}^{1}\right|^{2}$ are also zero mean complex Gaussian and exponential variables with average given by $\mu_{2}=\left|S_{k, l}^{p, p i l o t}\right|^{2} E\left[\left|H_{k, l}^{p, 1}\right|^{2}\right]+\mathrm{N}_{0}$, respectively. However they are not necessarily uncorrelated for different $\mathrm{k}$ and $\mathrm{l}$. Since the receiver does not have a priori knowledge about the PDP (Power Delay Profile) of each user while it is still detecting them it does not know the correlation between different channel frequency response coefficients. For that reason, we opted to employ a threshold located in the middle of those obtained assuming two extreme cases: uncorrelated channel frequency response coefficients and constant channel frequency response coefficients.

\subsection{Uncorrelated channel frequency response}

If the different channel frequency response coefficients, $H_{k, l}^{p, 1}$, can be assumed uncorrelated for different $k$ and $l$ (for example a severe time-dispersive channel) then the decision variable $Y_{p}$ will correspond to a sum of uncorrelated exponential variables resulting again in an Erlang random variable described by the following PDF

$$
p_{2}(y)=\frac{y^{N_{\text {pilots }}-1} \exp \left(-\frac{y}{\mu_{2}}\right)}{\mu_{2}^{N_{\text {pilots }}}\left(N_{\text {pilots }}-1\right) !}
$$

Therefore, the intersection of PDFs (16) and (17) results in the threshold given by

$$
y^{\text {th }}=\frac{N_{\text {pilots }} \ln \left(\frac{\mu_{2}}{\mu_{1}}\right)}{\frac{1}{\mu_{1}}-\frac{1}{\mu_{2}}}
$$

\subsection{Constant channel frequency response}

If the channel is basically non time dispersive then the channel frequency response coefficients, $H_{k, l}^{p, 1}$, will be almost constant for different $k$ and $l$ and, thus, the decision variable $Y_{p}$ will correspond to a sum of correlated exponential variables. To obtain the PDF for this case it is necessary to remind the fact that the exponential distribution is a special case of the gamma distribution. Consequently, we can employ the expression derived in (Aalo, 1995) for the sum of correlated gamma variables which, for this case, becomes

$p_{2}(y)=\frac{\left(\frac{y}{\mu_{2}}\right)^{N_{\text {pilots }}-1} \exp \left(-\frac{y}{(1-\sqrt{\rho}) \mu_{2}}\right){ }_{1} F_{1}\left(1, N_{\text {pilots }} ; \frac{\sqrt{\rho} N_{\text {pilots }} y}{(1-\sqrt{\rho})\left(1-\sqrt{\rho}+\sqrt{\rho} N_{\text {pilots }}\right) \mu_{2}}\right)}{\left(N_{\text {pilots }}-1\right) !(1-\sqrt{\rho})^{N_{\text {pilots }}-1}\left(1-\sqrt{\rho}+\sqrt{\rho} N_{\text {pilots }}\right) \mu_{2}} u(y)$

where $\rho$ is the correlation coefficient between different received samples which is constant and is defined as 


$$
\rho=\rho_{(k, l),\left(k^{\prime}, l^{\prime}\right)}=\frac{\operatorname{Cov}\left(\left|R_{k, l}^{1}\right|^{2},\left|R_{k^{\prime}, l^{\prime}}^{1}\right|^{2}\right)}{\sqrt{\operatorname{Var}\left(\left|R_{k, l}^{1}\right|^{2}\right) \operatorname{Var}\left(\left|R_{k^{\prime}, l^{\prime}}^{1}\right|^{2}\right)}},(k, l) \neq\left(k^{\prime}, l^{\prime}\right)
$$

with

$$
\operatorname{Cov}\left(\left|R_{k, l}^{1}\right|^{2},\left|R_{k^{\prime}, l^{\prime}}^{1}\right|^{2}\right)=2\left|S_{k, l}^{p, p i l o t}\right|^{4}\left(E\left[\left|H_{k, l}^{p, 1}\right|^{2}\right]\right)^{2}+2\left|S_{k, l}^{p, p i l o t}\right|^{2} E\left[\left|H_{k, l}^{p, 1}\right|^{2}\right] \mathrm{N}_{0}+\mathrm{N}_{0}{ }^{2}-\mu_{2}{ }^{2}
$$

and

$$
\operatorname{Var}\left(\left|R_{k, l}^{1}\right|^{2}\right)=2\left|S_{k, l}^{p, p i l o t}\right|^{4}\left(E\left[\left|H_{k, l}^{p, 1}\right|^{2}\right]\right)^{2}+4\left|S_{k, l}^{p, p i l o t}\right|^{2} E\left[\left|H_{k, l}^{p, 1}\right|^{2}\right] \mathrm{N}_{0}+2 \mathrm{~N}_{0}{ }^{2}-\mu_{2}{ }^{2}
$$

Alternatively, from (Alouini et al., 2001), we can also represent (19) as a single gamma-series

$$
p_{2}(y)=\frac{\lambda_{1}}{\lambda_{N_{\text {pilots }}}} \sum_{t=0}^{\infty} \frac{\delta_{t} y^{N_{\text {pilots }}+t-1} \exp \left(-\frac{y}{\lambda_{1}}\right)}{\lambda_{1}^{N_{\text {pilots }}+t}\left(N_{\text {pilots }}+t-1\right) !}
$$

with

$$
\delta_{t}=\left\{\begin{array}{l}
1, t=0 \\
\frac{1}{t} \sum_{i=1}^{t}\left(1-\frac{\lambda_{1}}{\lambda_{N_{\text {pilots }}}}\right)^{i} \delta_{t-i}, \quad t>0
\end{array}\right.
$$

and

$$
\lambda_{1}=\mu_{2}(1-\sqrt{\rho}) ; \lambda_{N_{\text {pilots }}}=\mu_{2}\left[1+\sqrt{\rho}\left(N_{\text {pilots }}-1\right)\right]
$$

${ }_{1} F_{1}(\cdot, ; \cdot)$ is the confluent hypergeometric function (Milton \& Stegun, 1964). The weighted intersection of PDFs (16) and (19) or (23) (threshold $y^{\text {th }}$ ) can be easily found numerically.

\section{Medium access control}

To evaluate the detection technique presented above we will use the analysis presented in (3GPP TR101 102 v3.2.0, 1998) for the network-assisted diversity multiple access (NDMA) MAC protocol. It is assumed that the users transmit packets to a BS, which is responsible for running most of the calculations and to handle transmission collisions. The BS detects collisions and uses a broadcast control channel to send a collision signal, requesting the users to resend the collided packets the required number of times $(p-1$ for a collision of $p$ packets). The remaining section studies how the throughput is influenced by the block/packet error rate (BLER), and compares the results with the performance of a contention-free scenario, based on TDMA. 


\subsection{Throughput analysis}

Following the NDMA throughput analysis of (3GPP TR101 102 v3.2.0, 1998), we consider a sequence of epochs where epoch is an empty slot or a set of slots where users send the same packet due to a BS request. Denoting $P_{e}$ as the probability of a user's buffer being empty at the beginning of an epoch, the binomial expressions for the probability of the epoch length for $J$ users are

$$
P_{b u s y}(p)=\left(\begin{array}{l}
J \\
p
\end{array}\right)\left(1-P_{e}\right)^{p} P_{e}^{J-p}, p=1,2, \ldots, J
$$

for a busy epoch and

$$
P_{\text {idle }}(p)= \begin{cases}P_{e}^{J}, & p=1 \\ 0, & p \neq 1\end{cases}
$$

for an idle epoch. The probability of having a useful epoch is

$$
P_{\text {usefull }}(p)=\left(\begin{array}{l}
J \\
p
\end{array}\right)\left(1-P_{e}\right)^{p} P_{e}^{J-p} P_{D}(p)^{p}
$$

where $P_{D}(p)$ is the frame's correct detection probability (equal to $1-B L E R$ ) when $p$ users are transmitting. We assume that no detection errors occur in the determination of the number of senders colliding. Finally, the throughput can be defined as

$$
R_{N D M A}=\frac{\text { average length of useful epoch }}{\text { average length of busy or idle epoch }}
$$

By using (26) and (29), and after some simplifications, we can write

$$
R_{N D M A}=\frac{\sum_{p=1}^{J} p\left(\begin{array}{c}
J-1 \\
p-1
\end{array}\right)\left(1-P_{e}\right)^{p} P_{e}^{J-p} P_{D}(p)^{p}}{J\left(1-P_{e}\right)+P_{e}^{J}}
$$

\subsection{Queue analysis}

If there are no detection errors at the receiver (i.e., the BS), then the busy and idle epochs have the distributions described by

$$
P_{\text {busy }}(p)=\left(\begin{array}{c}
J-1 \\
p-1
\end{array}\right)\left(1-P_{e}\right)^{J-1} P_{e}^{J-p}, 1 \leq p \leq J
$$

and

$$
P_{\text {idle }}(p)=\left\{\begin{array}{c}
P_{e}^{J-1}+(J-1)\left(1-P_{e}\right) P_{e}^{J-2}, p=1 \\
\left(\begin{array}{c}
J-1 \\
p
\end{array}\right)\left(1-P_{e}\right)^{p-1} P_{e}^{J-p-1}, 1 \leq p \leq J-1
\end{array}\right.
$$


where $P_{e}$ is the unique solution on [0,1] of the equation (see (3GPP TR101 102 v3.2.0, 1998))

$$
\lambda P_{e}^{J}+(1-\lambda J) P_{e}-(1-\lambda J)=0
$$

\subsection{Delay analysis}

For an $\mathrm{M} / \mathrm{G} / 1$ queue with vacation the average system delay for a data packet can be expressed as

$$
D=\overline{h_{\text {busy }}}+\frac{\lambda \overline{h_{\text {busy }}^{2}}}{2\left(1-\lambda \overline{h_{\text {busy }}}\right)}+\frac{\lambda \overline{h_{\text {idle }}^{2}}}{2 \overline{h_{\text {idle }}}},
$$

where $\overline{h_{b u s y}}, \overline{h_{b u s y}^{2}}, \overline{h_{\text {idle }}}$ and $\overline{h_{\text {idle }}^{2}}$ are the first and second moments of the busy and idle epoch respectively.

\subsection{Comparison with ideal TDMA protocols}

Traditional MAC protocols loose packets involved in collisions. The best performance with traditional MAC protocols is achieved when collisions are avoided, with a TDMA (time division multiple access) approach. The throughput for an ideal TDMA protocol depends linearly with the total offered load, and with the probability of correct detection of a single sender, i.e.,

$$
R_{T D M A}=\lambda J P_{D}(1)
$$

For large SNR $P_{D} \approx 1$ and (30) can be written as

$$
R_{N D M A}=\frac{J\left(1-P_{e}\right)}{J\left(1-P_{e}\right)+P_{e}^{J}} .
$$

It can be shown that (36) is equal to $R_{T D M A}$ when a Poisson source is used (see (3GPP TR101 102 v3.2.0, 1998). Therefore, NDMA and TDMA throughputs are the same when no detection errors occur, and converge to one near saturation. However, NDMA outperforms TDMA for low signal to noise ratio values, due to the detection gain for multiple transmissions.

\section{Numerical results}

In this section we present several performance results concerning multipacket detection for OFDM-based systems. The channel impulse response is characterized by the PDP (Power Delay Profile) based on the Vehicular A environment (3GPP TR101 102 v3.2.0, 1998), although similar results would be obtained for other severely time-dispersive channels. Rayleigh fading was admitted for the different paths. The number of subcarriers employed was $N=256$ with a spacing of $15 \mathrm{kHz}$ and each carrying a QPSK data symbol. The channel encoder was a rate- $1 / 2$ turbo code based on two identical recursive convolutional codes characterized by $G(D)=\left[1\left(1+D^{2}+D^{3}\right) /\left(1+D^{2}+D^{3}\right)\right]$. A random interleaver was employed within the turbo encoder. The coded bits were also interleaved before being mapped into a 
QPSK constellation. Each information stream was encoded with a block size of 3836 bits which, combined with a pilot insertion spacing of $\Delta N_{F}=N_{p, \max }$ and $\Delta N_{T}=16$ results in a frame composed of 16 OFDM blocks. The power level of the pilots symbols was chosen as $E\left[\left|S_{k, l}^{p, \text { Pilot }}\right|^{2}\right] / E\left[\left|S_{k, l}^{p}\right|^{2}\right]=10 \cdot \log _{10}\left(N_{p, \max }\right)(E[\cdot]$ represents the expected value computed over all positions $(k, l)$ containing pilot symbols in the case of the numerator and over all positions containing data symbols in the case of the denominator) so that the percentage of overall transmitted power spent on the pilots was always the same, independently of the maximum number of users, $N_{p, \max }$.

Regarding the channels for the $N_{P}$ retransmissions of a given packet, we considered three scenarios: uncorrelated channels (UC), fixed channels (FC), corresponding to a stationary scenario, and variable channels (VC), where the mobile speeds are $v=30 \mathrm{~km} / \mathrm{h}$. Unless otherwise stated, uncorrelated symbol interleavers are assumed for different retransmissions. The performance results are expressed as a function of the $E_{b} / N_{0}$, where $E_{b}$ is the average bit energy per packet and $N_{0}$ the one-sided power spectral density of the channel noise.

As explained previously, ideally the multipacket separation should be made using MLSO, but an MMSE-based separation is much less complex, especially for larger constellations and/or when a large number of packets collide. These multipacket separation techniques can be combined with IC in an iterative receiver as explained in Section 4 .

In Fig. 5 we present the BLER for MMSE and MLSO packet separation schemes with or without IC in the case of a collision involving 2 packets. For the schemes without IC we assumed that there are 12 iteration of the turbo decoder. For the cases with IC we have an initial MLSO/MMSE packet separation step and 3 IC steps, each one with 3 iterations applied inside the channel decoder. Regarding the retransmissions, we assumed the VC scenario (similar conclusions could be drawn for other scenarios). From this figure, it is clear that the best performances are attained when we combine MMSE or MLSO separation with IC (in fact, similar performances are achievable when MMSE or MLSO separation are combined with IC); if we do not employ IC an initial MLSO packet separation allows much better performance than MMSE packet separation). In the following results we will always assume an MMSE packet separation combined with 3 IC steps.

The figure (Fig 6) shows the impact of the symbol interleaving for different numbers of colliding packets. Four retransmission scenarios (VC without interleaving, FC and VC employing different symbol interleavers, and UC) are considered. Regarding the two VC scenarios, it is clear that, for the adopted mobile speeds $(30 \mathrm{~km} / \mathrm{h})$ the channel correlations are too high to allow efficient packet separation if we do not employ different symbol interleavers for different retransmissions. Comparing all the different scenarios, as expected, the performances are better for UC scenarios and worse for FC scenarios, with VC having performances in-between. It is important to highlight the fact that although the FC scenario corresponds to a channel that remains fixed for the retransmissions we can still achieve reliable detection with our receiver due to use of the symbols interleavers in the retransmissions.

In Fig. 7 we show the BLER performance for different values of $N_{p}$ assuming VC scenario. Clearly, our receiver allows an efficient packet separation. From this figure we can observe performance improvement as we increase $N_{p}$, which is a consequence of having adopted the $E_{b}$ for each packet (the total energy used to transmit a packet is $N_{p} E_{b}$, since the total number of versions that were transmitted is $N_{p}$ ). 


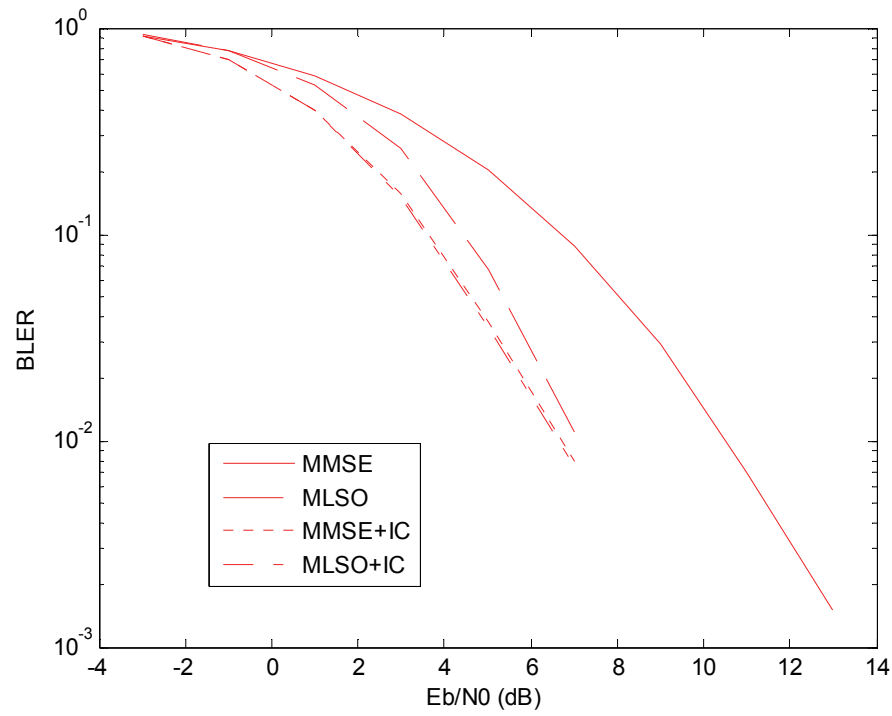

Fig. 5. BLER performance for different packet separation techniques, when $\mathrm{Np}=2$ for VC.

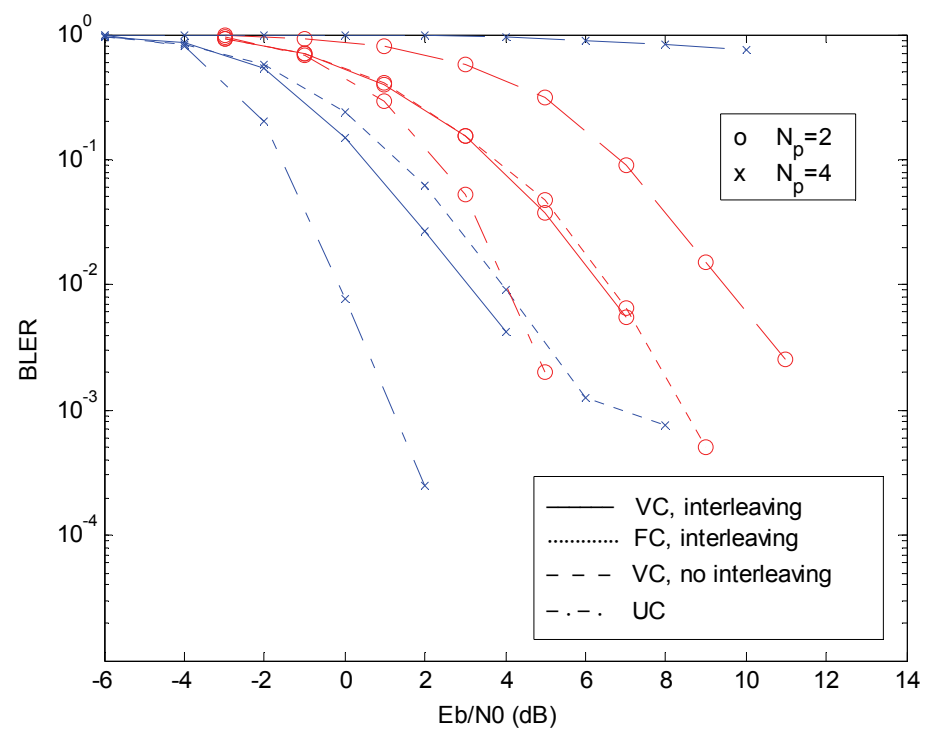

Fig. 6. Impact of using different interleavings for different retransmissions. 


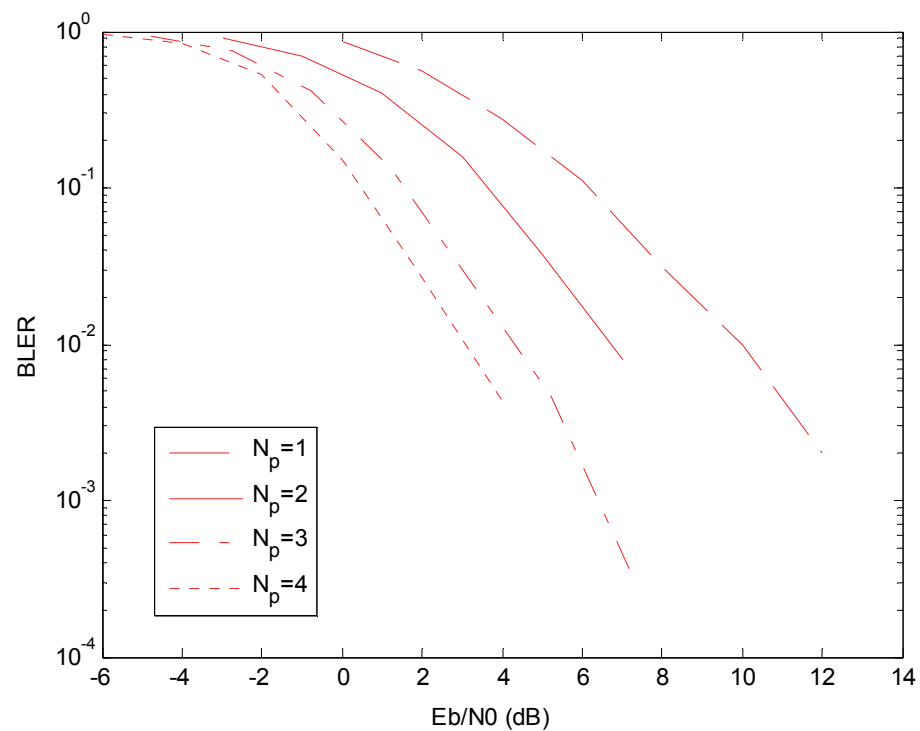

Fig. 7. BLER with different values of $\mathrm{Np}$ for VC.

Using the approach described in Section !!0!! we present the results regarding the Detection Error Rate (DER) for $N_{p, \max }=4$ and $\mathrm{P}_{\mathrm{e}}=0.2$ (a high probability of transmission for each user) in Fig. 8 .

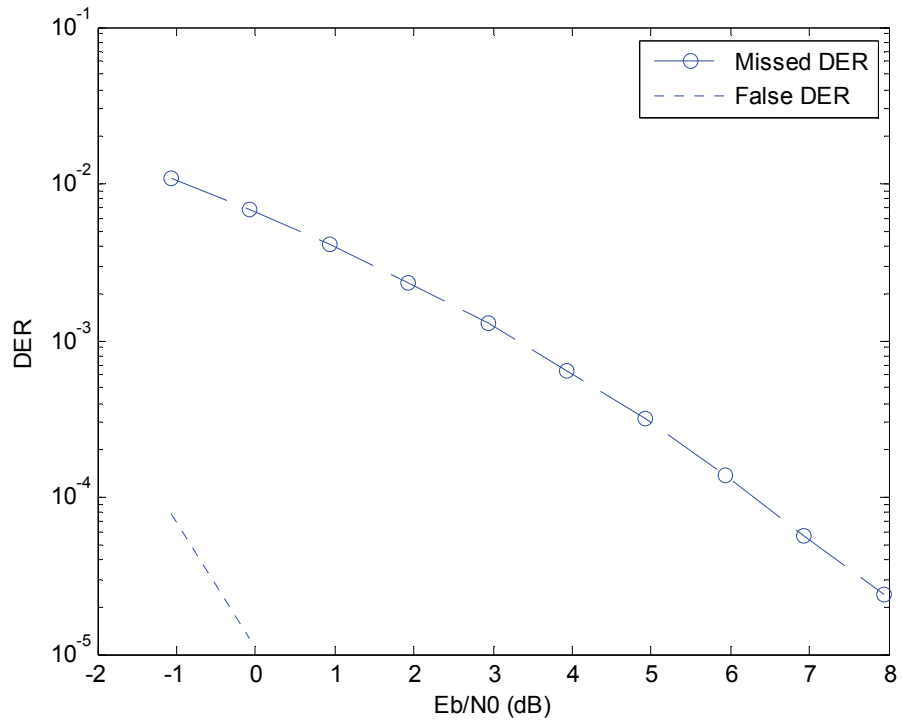

Fig. 8. Detection Error Rate for $\mathrm{Np}, \max =4$ and $\mathrm{Pe}=0.2$. 
Curves representing the false DER (false detection of users) and missed DER (users not detected) are shown. It is visible that for $E_{b} / N_{0}=2 \mathrm{~dB}$ that the DER is mostly caused by undetected users (the receiver cannot distinguish them from noise) with an error rate between $0.2-0.3 \%$ while false alarms are virtually inexistent.

Next we compare NDMA and TDMA throughputs for the scenario simulated previously. Throughput is calculated as described in Section 5, using BLER obtained above (it should be emphasized that our throughput model does not take into account invalid detection of the number of senders on a collision).

In Fig. 9, Fig. 10 and Fig. 11 show how $R_{N D M A}$ and $R_{T D M A}$ depend on the offered load, for $E_{b} / N_{0}$ values of $2 \mathrm{~dB}, 4 \mathrm{~dB}$ and $6 \mathrm{~dB}$, respectively. The offered load $(\lambda J)$ varies from very light load $(10 \%)$ until the saturation value $(100 \%)$, where all bandwidth is required to

satisfy the offered load. Results show that NDMA clearly outperforms TDMA for the conditions tested, especially for loads above $60 \%$, with higher differences for lower $E_{b} / N_{0}$. The reason for this behavior is that our receiver can take full advantage of the overall energy spent to transmit the packet (i.e., the energy for all retransmission attempts). Therefore, the performance of transmitting with success a given packet when we have a collision of several packets is higher than without collisions (as in the TDMA case) due to the BLER performance improvement with larger $N_{P}$ (as shown in Fig. 7). The only case where our technique is worse than conventional TDMA schemes is for slow-varying channels without symbol interleaving, especially for large system loads, since the correlation between different retransmissions can be very high, precluding an efficient packet separation.

The throughput obtained in fixed or variable channels combined with interleaving is only slightly worse than that obtained in uncorrelated channels.

We would like to point out that although the throughput for high system load can be close to $100 \%$, the corresponding packet delay grows fast for large system loads, since the number of retransmission increases with the number of collisions, and the number of collisions is higher for higher system loads.

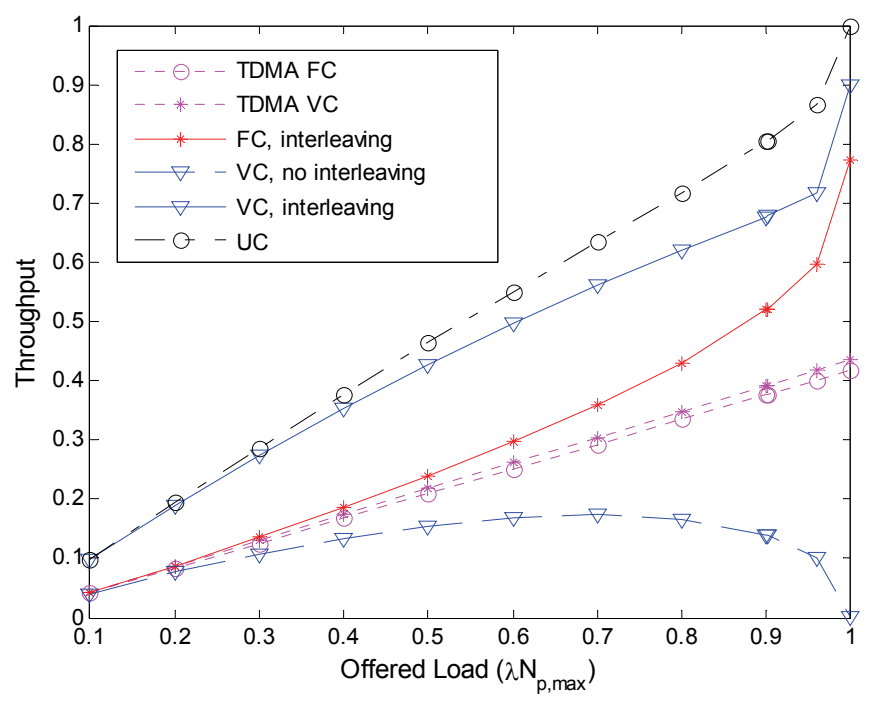

Fig. 9. Throughput when $\mathrm{Eb} / \mathrm{N} 0=2 \mathrm{~dB}$. 


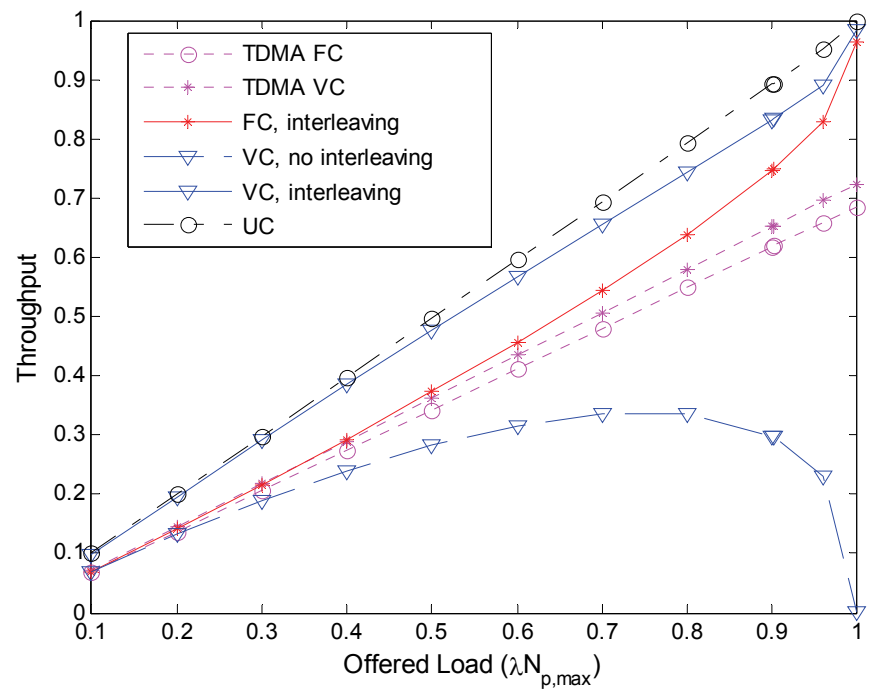

Fig. 10. Throughput when $\mathrm{Eb} / \mathrm{N} 0=4 \mathrm{~dB}$.

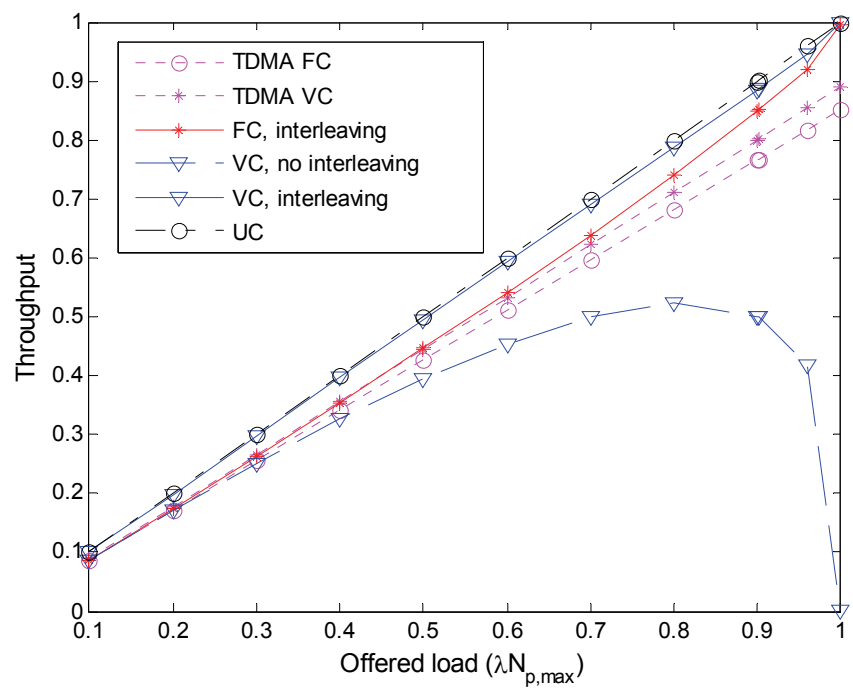

Fig. 11. Throughput when $\mathrm{Eb} / \mathrm{N} 0=6 \mathrm{~dB}$. 


\section{Conclusions}

In this chapter we considered a multipacket detection technique to cope with MAC collisions in OFDM-based systems. This technique allows high throughputs, since the total number of transmissions can be equal to the number of packets involved in the collision.

Since our packet separation technique requires different channels for different retransmissions we proposed the use of different interleavers for different retransmissions. This allows good performances even slow-varying channels. In fact, we can an efficient packet separation even when the channel remains fixed for all retransmissions. We also included a method to estimate the number of users involved in a collision, as well as the corresponding channel characteristics.

\section{Acknowledgments}

This work was partially supported by the FCT - Fundação para a Ciência e Tecnologia (pluriannual funding and U-BOAT project PTDC/EEA-TEL/67066/2006), and the CMOBILE project IST-2005-27423.

\section{References}

3GPP. 25.212-v6.2.0 (2004) “Multiplexing and Channel Coding (FDD),2004 , 3rd Generation Partnership Project, Sophia- Antipolis, France.

3GPP. TR25.814 (2006). "Physical Layers Aspects for Evolved UTRA".,2006 ,3rd Generation Partnership Project, Sophia- Antipolis, France.

3GPP TR101 112 v3.2.0 (1998). 'Selection procedures for the choice of radio transmission technologies of UMTS'., 1998 , 3rd Generation Partnership Project, SophiaAntipolis, France.

Aalo, V. A. (1995). "Performance of maximal-ratio diversity systems in a correlated Nakagami-fading environment". IEEE Trans. Commun., vol. 43, pp. 2360-2369, 0090-6778.

Abramowitz, M., \& Stegun, I. A. (1964). Handbook of Mathematical Functions with Formulas, Graphs, and Mathematical Tables. Dover Publications, 0-486-61272-4, New York, USA

Alouini, M. -S.; Abdi, A. \& Kaveh, M. (2001). "Sum of Gamma Variates and Performance of Wireless Communication Systems Over Nakagami-Fading Channels". IEEE Trans. On Veh. Tech., pp. vol. 50, no. 6, pp.1471-1480, 2001, 1751-8628.

Cavers, J. K. (1991). “An analysis of Pilot Symbol Assisted Modulation for Rayleigh Fading Channels". IEEE Trans. On Veh. Tech., vol. 40, no. 4, pp.686-693, November, 1991, 0018-9545.

Cimini, L. (1985). “Analysis and Simulation of a Digital Mobile Channel using Orthogonal Frequency Division Multiplexing". IEEE Trans. on Comm, Vol. 33, No. 7, pp.665-675 ,July , 1985.

Dinis, R.; Carvalho; P., Bernardo; L., Oliveira; R., Serrazina, M. \& Pinto, P. (2007). " Frequency-Domain Multipacket Detection: A High Throughput Technique for SCFDE Systems ". IEEE GLOBECOM'07., pp.4619-4624, 978-1-4244-1043-9, Washington DC., USA, December 2007. 
Dinis, R.; Serrazina, M., \& Carvalho, P. (2007). "An Efficient Detection Technique for SC-FDE Systems with Multiple Packet Collisions", IEEE ICCCN'07, pp.402-407 , ,Turtle Bay, USA, September 2007.

Kay, M. S. (1993). Fundamentals of Statistical Signal Processing: Estimation Theory, PrenticeHall, 0-13-3457117, Englewood Cliffs.

Kim, Y. -S.; Kim, C. -J.; Jeong, G. -Y.; Bang, Y. -S.; Park, H. -K. \& Choi, S. S. (1997). “New Rayleigh fading channel estimator based on PSAM channel sounding technique". IEEE International Conf. on Comm. ,Vol. 3 , pp.1518-1520., 0-7803-3925-8 Montreal, Canada , June 1997.

Nee, R. van \& Prasad, R. (2000). “OFDM for Wireless Multimedia Communications”. Artech House, 978-0890065303, Norwood, MA, USA.

Souto, N.; Correia, A.; Dinis, R.; Silva, J. C. \& Abreu, L. (2008). “Multiresolution MBMS transmissions for MIMO UTRA LTE systems". IEEE International Symposium on Broadband Multimedia Systems and Broadcasting., pp.1-6, 978-1-4244-1648-6, Las Vegas, USA, June 2008.

Tsatsanis, M.; Zhang, R. \& Banerjee, S. (2000). Network-Assisted Diversity for Random Access Wireless Networks, IEEE ,48, 3,Mar. 2000, pp.702-708, 1053-587X

Valenti, M. C. (2001). "Iterative Channel Estimation and Decoding of Pilot Symbol Assisted Turbo Codes Over Flat-Fading Channels". IEEE Journal on Selected Areas in Communications,Vol. 19 ,No. 9 ,Setember 2001 , pp. 1697-1705.

Wang, X.; Yu, Y. \& Giannakis, G. (2005). “A Roboust High-Throughput Three Algorithm Using Successive Interference Cancellation". IEEE GLOBECOM'05.,Vol.6, pp. 3601, St. Louis, USA, 0-7803-9414-3.

Yu, Y. \& Giannakis, G. (2005). “SICTA: A 0.693 Contention Tree Algorithm Using Successive Interference Cancellation”. IEEE INFOCOM'05. ,Vol. 3 , 1908 - 1916,March, 2005, 0743-166X

Zhang, R. \& Tsatsanis, M. (2002). "Network-Assisted Diversity Multiple Access in TimeDispersive Channels". IEEE Trans. On Comm., Vol. 50, No. 4, pp. 623-632.,April 2002, 0090-6778 


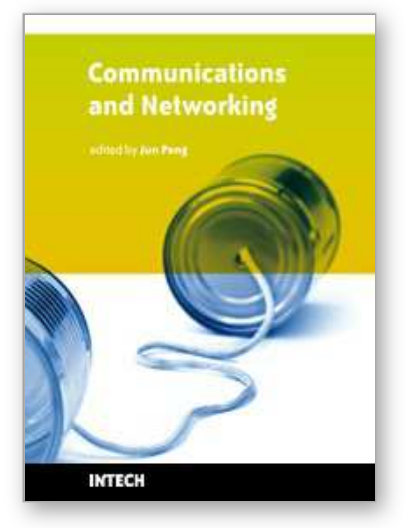

\section{Communications and Networking \\ Edited by Jun Peng}

ISBN 978-953-307-114-5

Hard cover, 434 pages

Publisher Sciyo

Published online 28, September, 2010

Published in print edition September, 2010

This book "Communications and Networking" focuses on the issues at the lowest two layers of communications and networking and provides recent research results on some of these issues. In particular, it first introduces recent research results on many important issues at the physical layer and data link layer of communications and networking and then briefly shows some results on some other important topics such as security and the application of wireless networks. In summary, this book covers a wide range of interesting topics of communications and networking. The introductions, data, and references in this book will help the readers know more abut this topic and help them explore this exciting and fast-evolving field.

\section{How to reference}

In order to correctly reference this scholarly work, feel free to copy and paste the following:

Nuno Souto, Rui Dinis, João Carlos Silva, Paulo Carvalho and Alexandre Lourenço (2010). High Throughput Transmissions in OFDM Based Random Access Wireless Networks, Communications and Networking, Jun Peng (Ed.), ISBN: 978-953-307-114-5, InTech, Available from:

http://www.intechopen.com/books/communications-and-networking/high-throughput-transmissions-in-ofdmbased-random-access-wireless-networks

\section{INTECH}

open science | open minds

\section{InTech Europe}

University Campus STeP Ri

Slavka Krautzeka 83/A

51000 Rijeka, Croatia

Phone: +385 (51) 770447

Fax: +385 (51) 686166

www.intechopen.com

\section{InTech China}

Unit 405, Office Block, Hotel Equatorial Shanghai

No.65, Yan An Road (West), Shanghai, 200040, China

中国上海市延安西路65号上海国际贵都大饭店办公楼 405 单元

Phone: +86-21-62489820

Fax: +86-21-62489821 
(C) 2010 The Author(s). Licensee IntechOpen. This chapter is distributed under the terms of the Creative Commons Attribution-NonCommercialShareAlike-3.0 License, which permits use, distribution and reproduction for non-commercial purposes, provided the original is properly cited and derivative works building on this content are distributed under the same license. 